\title{
Pulmonary Function Tests
}

By Dr. L. D. ERASMUS, M.B., B.Ch. (Rand), M.D. (Pretoria).

Member of the Faculty of Medicine, Pretoria University.

A Lecture given to Delegates and Members of the South African Society of Physiotherapy on the first day of the

"Chest Course", which was held at the Pretoria Hospital Physiotherapy School on May 27th and 28th, 1960.

DULMONARY function tests are of considerable value $P$ is assessing the physiological state of the lungs and can be used in many instances as a diagnostic tool. Frequently, however, it is used to assess the degree of disability and in other instances to assess progress made with treatment. Speciflc diagnostic problems include the investigation of cases of polycythaemia, obesity and the hypoventilation syndrome. They are sometimes used also to assess the efficiency of the respiratory muscles. It is convenient to consider pulmonary function tests under the headings of ventilatory and respiratory function.

\section{Ventilatory Function.}

The basic apparatus required is a spirometer with a recording drum and a method for assessing residual volume by a gas mixing technique. Before proceeding and further, however, I would like to remind the audience of the basic divisions of lung volume according to the so-called "New" Terminology of Pappenheimer, introduced in 1950.

Simple spirometric measurements can give considerable information in a matter of minutes. Thus all the divisions of total lung capacity except the residual volume can be determined but the residual volume has to be indirectly determined by a gas mixing technique.

\begin{tabular}{|c|c|c|c|}
\hline $\begin{array}{c}\text { TOTAL } \\
\text { lung } \\
\text { capacity }\end{array}$ & $\begin{array}{c}\text { Vital } \\
\text { capacity }\end{array}$ & $\begin{array}{c}\text { Inspiratory } \\
\text { reserve }\end{array}$ & $\begin{array}{c}\text { Inspiratory } \\
\text { capacity }\end{array}$ \\
\begin{tabular}{c|c|c|} 
Tidal volume \\
Expiratory \\
Reserve \\
volume
\end{tabular} & $\begin{array}{c}\text { Functional } \\
\text { Residual } \\
\text { Capacity }\end{array}$ \\
\hline
\end{tabular}

Vital Capacity.

Determination of the vital capacity is frequently valuable but has a limited application. Considerably more value is introduced if a time factor is also considered. For example the vital capacity in empysema and bronchial spasm may be normal but if the 2-second vital capacity is measured, a considerable reduction is usually found. Determination of the timed vital capacity after the renewal of spasm using a broncho-dilator drug is also valuable in distinguishing bronchial spasm from permanent organic change. In general it can be said that restrictive pulmonary lesions

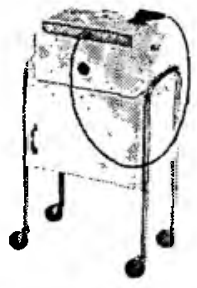

\section{THE BIRTCHER MEGASON VI ULTRASONIC UNIT}

To the more than 20,000 physicians now using ultrasonic therapy in the treatment of a host of acute and chronic conditions, this precision instrument adds new dimensions of accuracy and treatment ease. Descriptives and medical journal reprints on request.
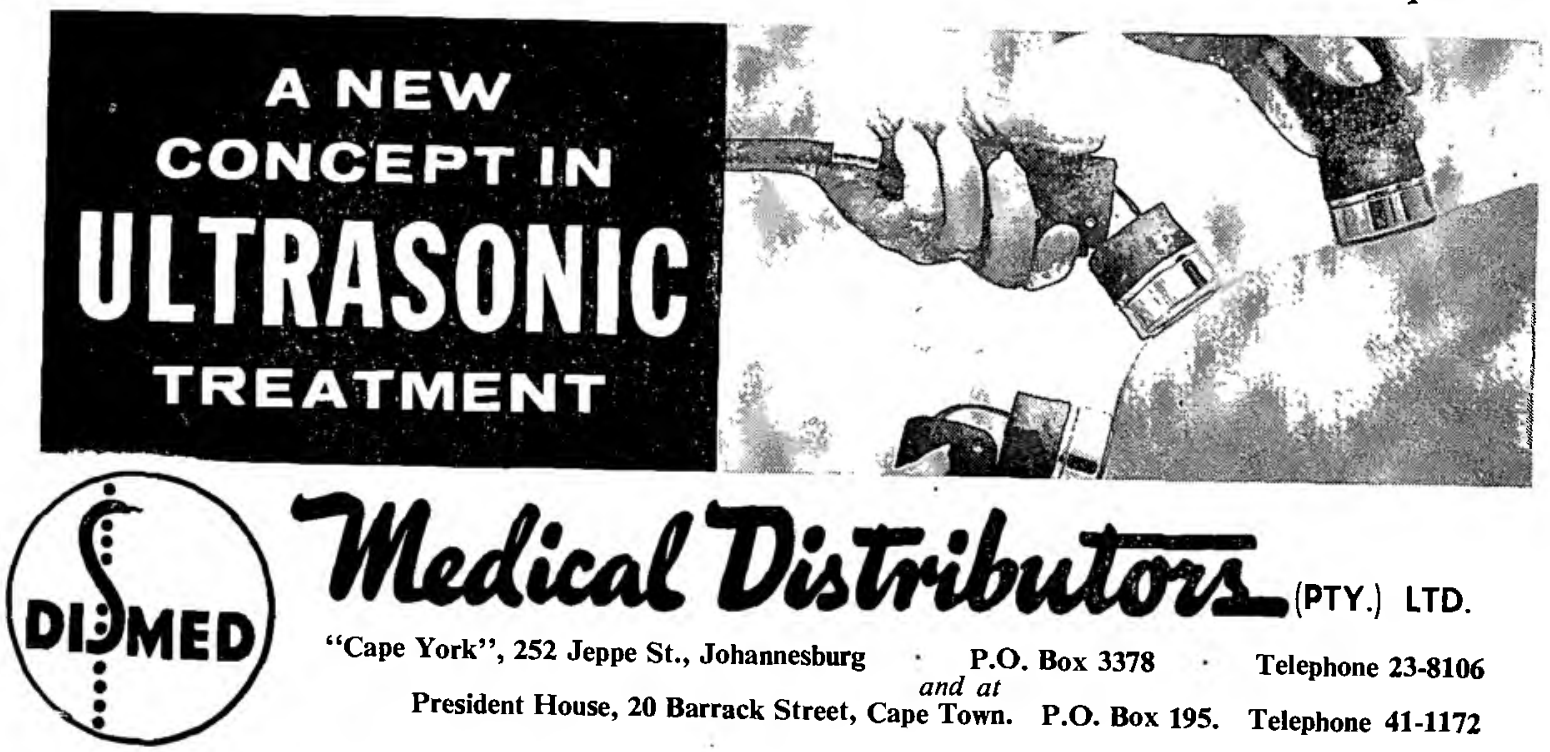

“Cape York", 252 Jeppe St., Johannesburg

President House, 20 Barrack Street, Cape Town 
reduce the vital capacity as such and obstructive pulmonary lesions reduce the timed vital capacity. In this fashion obstructive and restrictive lesions are easily distinguished and combinations of the two can be recognised.

The following illustrations show normal expiration with a timing device, then an expiration from a patient with an obstructive pulmonary lesion and then an expiration from a patient with a restrictive pulmonary lesion.

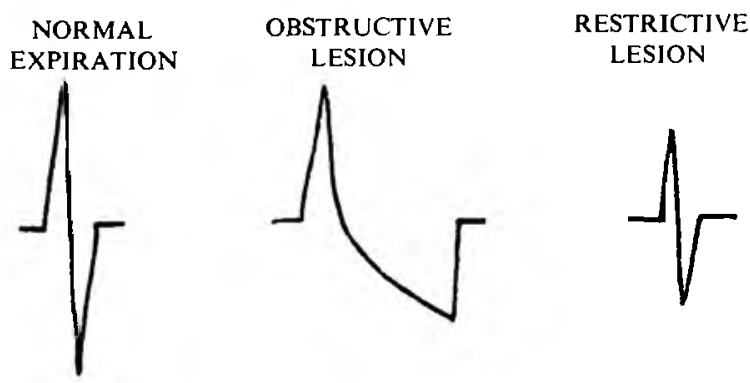

The curve of the obstructive lesion shows typical air trapping.

Maximum Breathing Capacity.

This is determined by getting the patient to breathe as rapidly and as deeply as possible while the respiratory excursions are recorded. A normally performed maximum breathing capacity will give a curve as follows and this is followed by another curve showing typical air trapping.
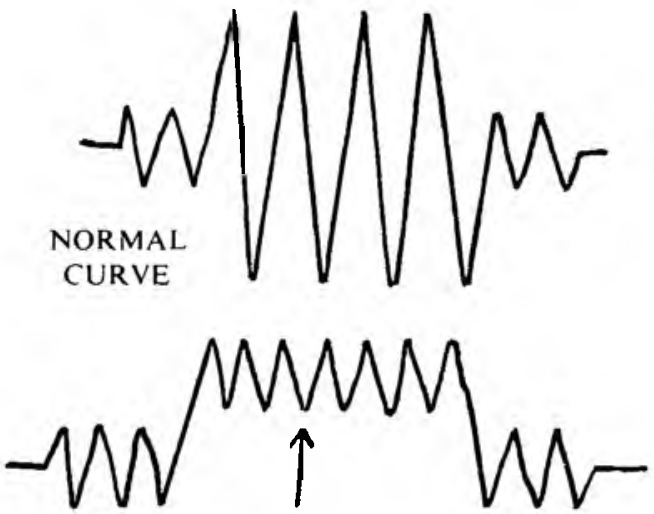

AIR TRAPPING

It is valuable to represent restrictive and obstructive lesions in the following fashion:

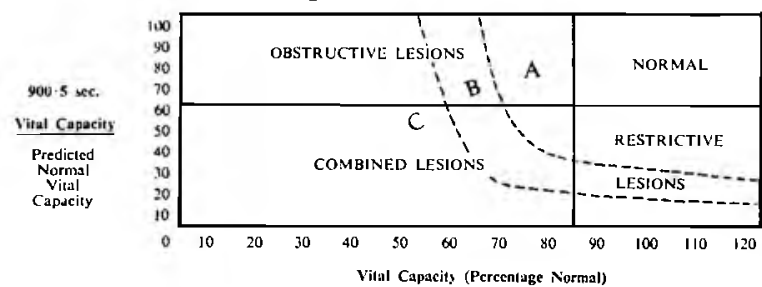

Using this method one can distinguish zones of disability and in the graph shown zone $A$ shows relatively normal patients, zone $B$ those with a moderate degree of pulmonary disability and zone $\mathrm{C}$ gross disability. This method has also been used for assessing the degree of operative risk for patients undergoing operation and those in group $\mathrm{C}$ are very poor risks. Those in group $B$ also are patients at risk and complications relating to the lungs are to be expected. It is particularly in this group where physiotherapy is likely to be of most value.
Additional information is given by various refinements of the pulmonary function tests.

Residual Volume.

This must be measured indirectly using an indicator gas usually helium. The indicator gas is measured and mixed with the patient's functional residual capacity which is then measured from the degree of dilution of the indicator gas. Intrapulmonary Mixing and Distribution of Gases.

Abnormalties here are found particularly in emphysema. The theory is that every breath taken is evenly distributed throughout normal lungs and this even distribution is grossly disturbed in patients with emphysema.

Elastance and Compliance.

This implies a measurement of the elastic properties (or lack of elastic properties) of the lung. This is measured with the assistance of an air-flled balloon in the oesophagus and depends on the physical principle of the degree of change of volume produced by change of pressure in the thorax. Changes in the compliance of the lung is found particularly in emphysema and in fibrosis of the lung.

Diffusing Capacity.

This measures the capacity of the alveolar membrane to allow gasses to pass through it to and from the arterial blood. This may be measured for oxygen and indicator gases such as carbon monoxide.

Blood Chemistry Changes.

These are of the greatest importance and the factors measured are the $\mathrm{pO}_{2}$ and $\mathrm{pCO}_{2}$ of the blood and of the alveolar air as well as the plasma bicarbonate.

Oximetry.

Arterial oxygen levels are measured before and after exercise and are found to fall considerably in patients having a defective diffusing capacity.

Bronchospirometry.

The function of the lungs may be measured separately using a special double-lumen tube which is passed into the left main bronchus which is obstructed with an inflatable balloon at the end of the tube. The oxygen uptake of the two lungs is measured separately.

Application of Pulmonary Function Tests.

\section{Diagnosis:}

Emphysema: Many cases of gross emphysema are easily diagnosed but it is sometimes quite surprising how the clinical and radiological assessment may be normal where pulmonary function tests show a considerable deviation from normal.

Total lung capacity is frequently nornal but residual volume is considerably increased. The other deviations include a marked reduction in timed vital capacity with air-trapping and delayed mixing of the gasses in the lungs. I Compliance is reduced and the maximum breathing capacity usually markedly reduced with an air-trapping phenomenon. Blood chemistry reveals an acidosis with increased plasma bicarbonate, reduction in the arterial oxygen level and an increase in the carbon dioxide level.

Fibrosis of the Lungs: Total lung capacity is frequently reduced and usually all divisions of lung volume partake in this reduction, thus the vital capacity is reduced and although the timed vital capacity is reduced, this is normal in proportion to the measured vital capacity. No air-trapping is found and mixing is usually delayed with a reduction of pulmonary compliance. Maximum breathing capacity is reduced but no air-trapping phenomenon is seen. Blood chemistry is usually normal except for a reduction of arterial oxygen levels. Tests for diffusing capacity of the lungs usually show a well-marked reduction.

Gross obesity: This is a very interesting phenomenon. All the divisions of pulmonary function are reduced but this especially applies to the tidal volume so that a considerably reduced minute volume occurs. Alveolar ventilation becomes so much reduced that arterial oxygen levels fall and the anoxaemia causes pulmonary hypertention, right ventricular hypertrophy and in some cases even cardiac failure and death from cor pulmonale. Secondary polycythaemia may 
occur. The interesting thing is that all these abnormalities can return to normal if the patient loses the excess weight. Hypoventilation Syndrome:

1. Pickwickian syndrome. This is very similar to the pattern produced by gross obesity and, in fact, gross obesity is one of the characteristics of this syndrome. In addition there is marked somnilence, cyanosis and polycythaemia. The syndrome derives its name, of course, from the fat boy of 'The Pickwick Papers' (Charles Dickens).

2. Damage to the respiratory centre. This may occur as a result of encephalitis and these patients may present with no pulmonary disability but with polycythaemia and cyanosis. Once again, the characteristic finding is that of alveolar hypoventilation.

Polycythaemia: These patients are frequently brought for pulmonary function tests with a view to deciding whether this is a primary deviation of the bone marrow or secondary to pulmonary pathology. For the most part, arterial oxygen levels are normal in primary polycythaemia and considerably reduced in polycythaemia due to pulmonary pathology. A full range of pulmonary function tests frequently provides the answer in this type of problem.

Assessment of Progress.

The degree of bronchospasm is easily estimated using some form of timed vital capacity or maximum breathing capacity and then comparing the results before and after a broncho-dilator drug. Periodic estimations also give a very good idea of long term therapy.

\section{Chronic Bronchitis and Emphysema}

$B y$ Dr. L. D. ERASMUS, M.B., B.Ch.(Rand)M.D. (Pretoria) Member of the Faculty of Medicine, Pretoria University.

A Lecture given to Delegates and Members of the South African Society of Physiotherapy on the first day of the "Chest Course", which was held at the Pretoria Hospital Physiotherapy School on May 27th and 28th, 1960.

Although either of these conditions may occur separately they are so frequently associated as almost to appear inseparable. An allergic factor or frank bronchial asthma is a frequent accompaniment. Chronic bronchitis and emphysema are conditions of paramount importance because of the high morbidity and even mortality brought about by acute exacerbations and complications. They occur frequently and result in tens of thousands of lost man-hours every year and treatment is frequently expensive, drawn out and usually requires hospitalization.

Clinical History.

Established chronic bronchitis and emphysema is usually preceded for several years by chronic cough which is usually productive and for several years before the pattern becomes established frequent attacks of acute winter bronchitis occur. Attacks of asthma are not infrequent. Later persistent exertional dyspnoea occurs and this may be interspersed with more acute attacks of bronchospasm.

The attacks of bronchitis may be suppurative in nature due to severe secondary infection and especially in the elderly disabling and even fatal attacks of broncho-pneumonia may occur. These attacks frequently precipitate congestive cardiac failure which also occurs particularly in the older age group when arteriosclerosis and hypertension have become established and have added another burden. Secondary polycythaemia frequently increases the blood viscosity and occasionally papiloedema and blindness may follow.

Aetiology.

External factors are frequently of the greatest importance and the incidence of chronic bronchitis is highest in those areas where there is pollution of the atmosphere by industrial gases and dust. Chronic bronchitis is also more frequent in heavy smokers. Pollution of the atmosphere is becoming a world wide problem. We breathe as much as $3 \pm$ Ibs. of air by weight in 24 hours, equal in weight to tin food we consume, but for many years it has not occurred to
Emphysema is usually permanent and frequently progressive and it is frequently only associated bronchospasm or infection that can be influenced by treatment. Pulmonary function tests have been studied before and after the application of physiotherapy to these patients and it appears that the degree of pulmonary function deviation is not influenced by physiotherapy.

Patients with pleurisy and pleural effusion show considerable improvement in their vital capacity as the condition improves.

Congestive cardiac failure. The degree of pulmonary oedema is indicated by a reduction in the vital capacity. In some instances pulmonary congestion can be determined at an early stage by measuring the vital capacity.

The progress of patients with weakness of the respiratory muscles can sometimes be effectively followed by doing the maximum breathing capacity test. This is repeated at short intervals and fatiguability will be indicated by progressive reduction in subsequent tests. A similar cluster of tests can be done at an interval of days or even weeks and progress followed in this fashion.

In conclusion it may be said that pulmonary function tests do not replace other methods of assessing the condition of the patient's lungs but they are in many instances a very valuable adjunct.

men to be as fastidious about the air they breathe as the food they eat. We will probably have to put up with grossly contaminated atmospheres for many years to come. Irritation from dust in dusty occupations such as mining is also of considerable importance.

An infective agency is important and the commonest infecting organism is $\mathbf{H}$. influenza but a mixed bacterial flora is frequently found. Individual predisposing factors include a hereditary tendency and allergy.

Basic Pathology.

It is convenient to consider the respiratory unit as consisting of a terminal and a respiratory bronchiole leading to the alveolar sacs. This may be represented in the following fashion in the normal
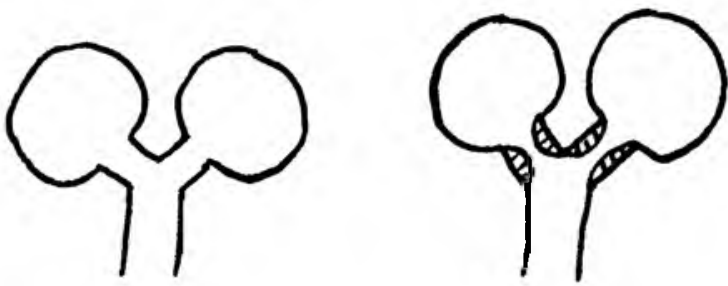

and thus when there is bronchial inflamation or spasm i.e., with an obstructive factor introduced.

With emphysematous dilation, rupture and coalescence of alveolar sacs occur as the result of degenerative changes in the elastic fibres of the alveolar walls. The factor of bronchial obstruction and infection is frequently superadded and there may be associated inter-alveolar fibrosis. These changes result in:-

1. A mechanical ventilatory deficiency where expiration especially is difficult due to bronchiolar obstruction, loss of elastic tissue and the patient having to expire from an initial level of hyperinflation.

2. Inefficient distribution of the air within the alveolar sacs occurs and the impaired diffusion of the gasses occurs.

3. In turn anoxaemia and carbon dioxide accumulation occurs in the blood resulting in acidosis and in compensatory increase of the blood bicarbonate.

In terms of pulmonary function tests the lung volume may remain the same (as will the vital capacity in many instances) but expiratory difficulty results in air trapping, 